\title{
Value of DermaCheckup as a novel dedicated digital health solution for teledermatology
}

\author{
PETRE C. ILIE ${ }^{1}$, SIMINA STEFANESCU ${ }^{2}$, JANE ALDRIDGE $^{3}$, \\ PHILIP GAFFNEY ${ }^{3}$, JAMES BELCHER ${ }^{3}$ and LEE SMITH ${ }^{4}$ \\ ${ }^{1}$ Department of Urology, Norfolk and Norwich University Hospital, Norwich NR4 7UY; ${ }^{2}$ Dermatology Department, \\ The Queen Elizabeth Hospital, King's Lynn PE30 4ET; ${ }^{3}$ L2S2 Ltd., Cambridge CB24 3DQ; \\ ${ }^{4}$ Centre for Health, Performance and Wellbeing, Anglia Ruskin University, Cambridge CB1 1PT, UK
}

Received September 22, 2021; Accepted January 10, 2022

DOI: $10.3892 / \mathrm{mi} .2022 .30$

\begin{abstract}
Daily healthcare is becoming increasingly costly and resource-intensive, requiring vast human and financial resources. The primary aim of the present study was to present the initial findings regarding the diagnostic accuracy of a novel telemedicine platform, DermaCheckup, when compared with face-to-face clinical appointments. The secondary aim was to assess whether patient management plans produced via this telemedicine platform differ from those decided upon following a face-to-face dermatological consultation. The difference in time to diagnosis between the tele-dermatology platform and standard care was also assessed. The DermaCheckup teledermatology service was implemented in March, 2020 as the COVID-19 pandemic emerged in the UK. The present study assessed patients who underwent a face-to-face clinical consultation, who, prior to visiting the clinic, used the teledermatology platform; thus, the diagnosis made via teledermatology was able to be compared to that made following standard care. Comparisons were made between diagnosis, process of diagnosis and the time to diagnosis. A total of 29 consecutive patients entering one UK dermatology clinic were included in the study. The COVID-19 pandemic resulted in face-to-face visits being challenging, owing to the risk of transmission of SARS-CoV-2. This limited the number of patients recruited into the study. The DermaCheckup application exhibited a very good level of agreement in terms of diagnosis with traditional face-to-face appointments. In the present study cohort, $93 \%$ of the patients could have been managed safely or referred immediately for a biopsy, thus avoiding a visit to the hospital. A substantial important improvement was also observed in the efficiencies
\end{abstract}

Correspondence to: Dr Petre C. Ilie, Department of Urology, Norfolk and Norwich University Hospital, Colney Ln, Norwich NR4 7UY, UK

E-mail: petre.ilie@nnuh.nhs.uk

Key words: dermatology, telemedicine, digital health, teledermatology, remote consultation, e-health, diagnosis that can be potentially achieved; the time to manage a patient decreased between 46-22-fold without considering the waiting time required between the time of organizing an appointment to the actual appointment.

\section{Introduction}

Daily healthcare is becoming increasingly costly and resource-intensive, requiring a vast number of resources, both human and financial (1). The use of healthcare services has increased over the recent decades, which is predominantly owing to an ageing population (2). This increase in the use of these services has placed considerable strain on the existing infrastructure. Thus, there is a need for the development and implementation of novel and efficient services for healthcare. Of note, a specific area of medicine in which an increasing service demand has been noted due to the ageing population, as aforementioned, is that of dermatology (3).

Due to this increasing demand for dermatology-related services, the present study demonstrates the use of a novel teledermatology platform. This is a digital health solution co-designed by medical practitioners, clinical leads (including dermatologists), IT specialists and operations management specialists aimed at improving the methods through which dermatological-related consultations are managed (Fig. 1).

The primary aim of the present study was to demonstrate the initial findings regarding the diagnostic accuracy of this telemedicine platform (DermaCheckup; L2S2 Ltd.) when compared with face-to-face clinical appointments. The secondary aim was to assess whether patient management plans produced via the telemedicine platform differ from those decided upon following a face-to-face dermatological consultation. In addition, the difference in time to diagnosis between the teledermatology platform and standard care was assessed.

\section{Materials and methods}

The DermaCheckup teledermatology service was implemented in March, 2020 as the COVID-19 pandemic emerged in the UK. The platform was designed and tested in the UK prior to this, with the initial design and planning lasting 2 years. For 1 month, during this present pilot trial, patients referred 
by a general practitioner, who underwent a face-to-face clinical consultation were assessed. The platform consists of a web-based interface for clinicians and an app-based one (also including the possibility of computer access) for patients. Prior to visiting the clinic, the patients used the teledermatology app; thus, the teledermatology diagnosis was made and compared to the one established using standard care. Comparisons were performed between the two types of care as regards diagnosis, the process of diagnosis and the time to diagnosis. The teledermatology platform integrated instructions of how to obtain images and algorithms for collecting information. (Fig. 1). From the initial iterations it was learnt that is it important to provide patients with instructions on how to obtain images in order to ensure they receive the maximum benefits from the platform. This is added as a short video. The algorithm is designed to request information based on the data already provided in order to optimise the diagnosis and at the same time to make the platform user-friendly and easy to use. Patients are asked about the location, different characteristics of the lesions, previous treatments, etc. Specifically, the app collects the type of information that would normally be obtained by the clinician. The patients can connect using a secure app and obtain images of their dermatological issue using their mobile camera or they can upload the information using a computer.

The platform was designed to advance the teledermatology process described by Giavina-Bianchi et al (4), as patients can use a mobile phone, and a user-friendly design, to complete the necessary information. This novel approach for the diagnosis and treatment of patients while in their homes would require a secure connection, storage and data handling technologies. The platform has acquired all appropriate NHS security certificates and meets NHS IT requirements. Moreover, the design is user friendly and considers the ability of the end user. Importantly, the application can be securely downloaded and installed by patients (iOS or Android). The application allows patients to obtain images of their skin condition using their mobile phones (videos can also be used as per the patient's preference). Moreover, there is an option to also use a web-based login for patients that do not own a smart phone or are not comfortable using the technology.

The information entered by the patient is subsequently reorganised to facilitate, review and enhance the efficiency for the diagnosing clinician. Using automation, the diagnosis and recommendations are straightforward and the time spent diagnosing is minimised. The diagnostic accuracy is also assisted by the instructions provided to the patients while obtaining the images.

In the present study, patient consent was obtained prior to the trial. Ethical approval has followed the local guidelines for clinical audits.

\section{Results}

A total of 29 consecutive patients (approximately one third of the total) entering the dermatology clinic at The Queen Elizabeth Hospital, were included in the present study. The COVID-19 pandemic resulted in face-to-face visits becoming challenging owing to the risk of the transmission of SARS-CoV-2. This limited the number of patients recruited into the study. The clinical diagnosis was found to be the same between DermaCheckup and the face-to-face consultation in 25 of the $29(86.2 \%)$ patients. For 3 patients (10.3\%), a certain diagnosis could not be established remotely and the clinical appointment diagnosis was the following: A typical pigmented lesion, dermatofibroma and blisters. For 1 patient, the remote diagnosis was that of dermatitis and the clinical appointment final diagnosis was that of lichen sclerosus. The pathology was diverse and complex; thus, the present study was able to provide a representative comparison between the diagnosis made by teledermatology and that by the face-to-face consultation for the majority of dermatological conditions (Table I).

The most commonly diagnosed conditions were the following: Basal cell carcinoma or squamous cell carcinoma (7 patients), benign mole (6 patients), seborrheic keratosis (3 patients) and atypical lesion (3 patients). Only 1 patient was diagnosed with melanoma (Table I).

The mean time required for diagnosis using telemedicine was only $26 \mathrm{sec}$ compared with the time required for a clinical appointment, which was $9.76 \mathrm{~min}$ (commencing from the point of arrival at the clinic).

\section{Discussion}

The value of using images in dermatological conditions for paediatric population was previously described by O'Connor et al (5) and the value of remote diagnosis in dermatology has been previously highlighted (4). For example, Giavina-Bianchi et al (4) found that $43 \%$ of patients required referral from primary care after using 'store-and-forward' teledermatology and that $53 \%$ were treated directly in primary care, with a further $4 \%$ being directly referred to biopsy. It is considered that by improving the design of the tools and the algorithm, this number can be decreased further. In the present study, only 3 patients with an unknown diagnosis required a face-to-face visit. In one case, the diagnosis of dermatitis made remotely was changed to that of lichen sclerosus. The lesions appear relatively similar and the use of dermoscopy in the clinical setting is used to establish the diagnosis. For this case, the disease management would have included the use of moisturisers being suggested remotely and the addition of topical steroids after the clinical assessment. It is considered that the management with moisturiser would have been safe as the follow-up would have detected the potential lack of improvement and triggered a different management. The remaining cases presented herein could have been managed remotely. This indicates that $93 \%$ of the patients could be treated remotely without the need for hospital visits.

The fact that the management of the majority of the patients in the present study not being altered following a face-to-face consultation suggests that this is a safe method for the assessment and management of skin conditions. For 1 patient, the diagnosis was changed following a clinical assessment. This is considered an acceptable clinical variation in diagnosis and arranging follow-up visits can prevent deterioration in the case of lichen sclerosus.

There are different types of platforms, such as the synchronous video and 'store and forward' platforms, each with advantages and disadvantages. The 'store and forward' has the most evidence for continuity of care for patients and also for triaging (teletriage) (6). For the synchronous video 


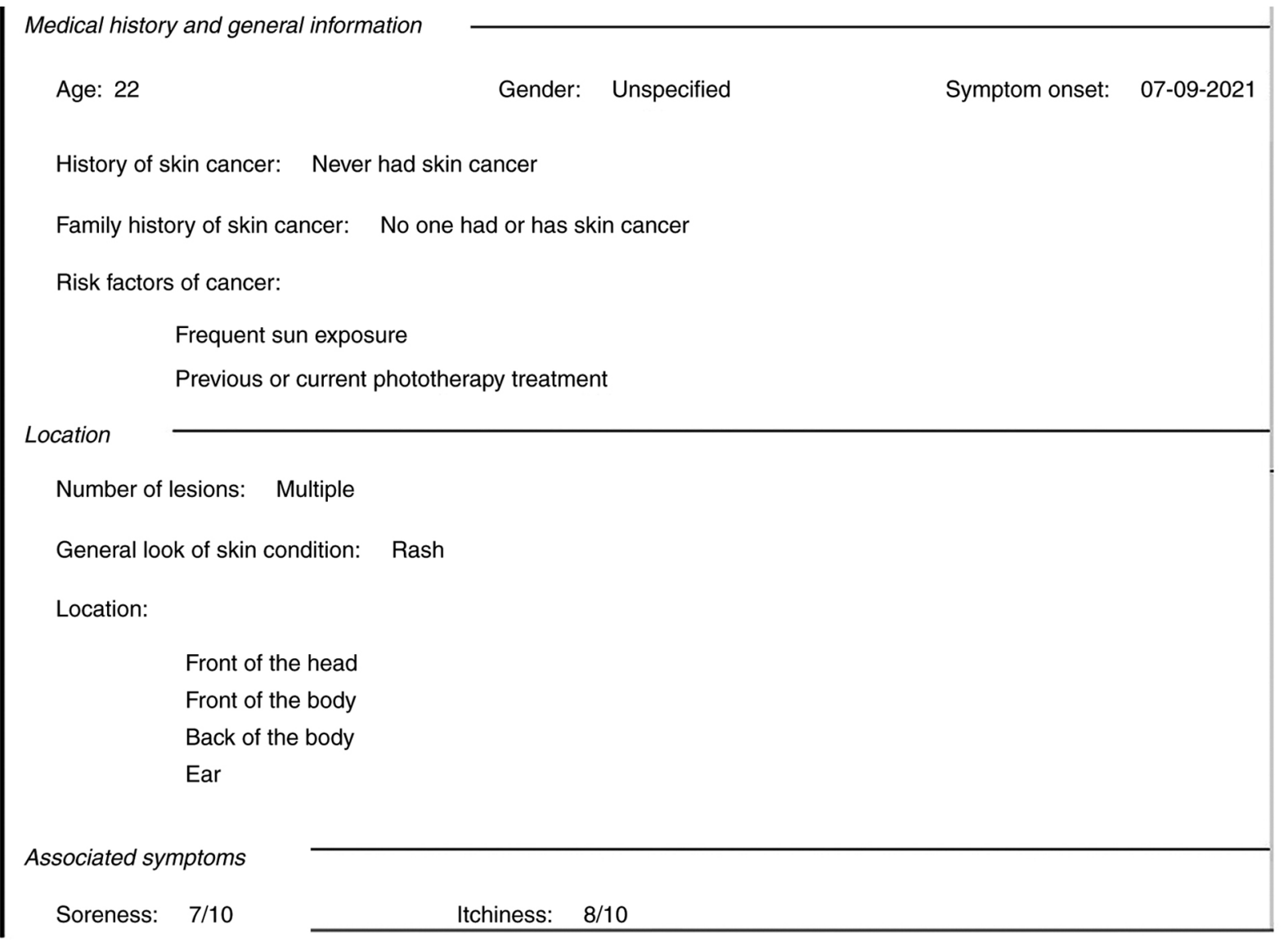

Figure 1. Example of the teledermatology platform. The platform integrates instructions of how to obtain images and algorithms for collecting information. The algorithm is designed to request information based on the data already provided in order to optimise the diagnosis and at the same time to make the platform user-friendly and easy to use. Patients are asked about the location, different characteristics of the lesions, previous treatments, so on and so forth. The patients can connect using a secure app and obtain images of their dermatological issue using their mobile camera or they can upload the information using a computer.

platform, the requirements for technology are higher for the hardware device (mobile phone, etc.) as well as for the internet bandwidth. This can lead to disparities in the access to health services. On the other hand, it can bring economies to multiple stakeholders. Patients will not need to travel to the hospital and wait for their clinical appointment. Assis Acurcio et al (7) demonstrated that teledermatology service has proven to be a cost-effective alternative to conventional dermatology, by reducing the costs associated with commuting. This may be an attractive alternative as the teledermatology solution can be used at a time convenient for the patient. The same applies for the clinician, as a practice may be able to manage patients more efficiently. Finally, such a type of medical practice is crucial for aiding in the timely diagnosis of patients during the current COVID-19 pandemic, as individuals may wish to avoid hospital visits or some hospital services may not be running. It would also provide an electronic record of the images and information that can be used at subsequent visits to objectively assess the result of the treatment. By reducing the time required for patient assessment, the teledermatology platform can also increase the efficiency of the clinicians who would have more time for specialist work. This may be particularly beneficial for private practice.

The use of this automated solution managed by dermatologists has led to a decrease in the time required for a diagnosis to be made, as well as for a prescription to be administered. In addition, it provides the patient with information within 26 sec. This is a marked improvement, as this is 22.5 times faster compared with the classical approach of $9.76 \mathrm{~min}$ in the current trial. Importantly, in current clinical practice, the time for face-to-face clinical consultation is efficient. Indeed, a number of dermatological clinics may have slots every 15-20 min for their patients, which will increase the time required to see the patients. In that case, the platform can deliver the diagnosis up to 46 times faster. Another important benefit of DermaCheckup is that it likely reduces the time to diagnosis as the patient does not have to physically book an appointment to see a consultant face-to-face, where a waiting time of $\geq 2$ weeks may be required. The authors plan to further develop this automation with artificial intelligence in order to improve the efficiency even further. Giavina-Bianchi et al (8) described the value of artificial intelligence, a deep neural network algorithm in triaging chronic skin conditions. They concluded that the algorithm was accurate in triaging, making a correct referral and also in prioritizing common chronic skin diseases to primary care attention (8). In the current platform, considering that dermatologists make decisions based on information available, this still involves clinician-driven patient management and the platform facilitates information collection in a patient-friendly manner; it also disseminates 
Table I. Remote vs. clinical diagnosis and management.

\begin{tabular}{|c|c|c|c|c|}
\hline $\begin{array}{l}\text { Patient } \\
\text { no. }\end{array}$ & $\begin{array}{l}\text { Remote } \\
\text { diagnosis }\end{array}$ & $\begin{array}{l}\text { Remote } \\
\text { management }\end{array}$ & $\begin{array}{l}\text { Final clinical } \\
\text { diagnosis }\end{array}$ & $\begin{array}{l}\text { Clinical } \\
\text { management }\end{array}$ \\
\hline 1 & Atypical lesion & Excision & Atypical lesion & Excision \\
\hline 2 & Unknown & Biopsy, blood tests & Unknown & biopsy, blood tests \\
\hline 3 & Angioma & Reassurance & Angioma & Reassurance \\
\hline 4 & Prurigo & Steroids, biopsy & Prurigo & Steroids, biopsy \\
\hline 5 & Seborrheic keratosis & $\begin{array}{l}\text { Reassurance, cryotherapy } \\
\text { (cosmetic) }\end{array}$ & Seborrheic keratosis & $\begin{array}{l}\text { Reassurance, cryotherapy } \\
\text { (cosmetic) }\end{array}$ \\
\hline 6 & Seborrheic keratosis & $\begin{array}{l}\text { Reassurance, for shave } \\
\text { (cosmetic) }\end{array}$ & Seborrheic keratosis & $\begin{array}{l}\text { Reassurance, for shave } \\
\text { (cosmetic) }\end{array}$ \\
\hline 7 & Basal cell carcinoma & cryotherapy & $\begin{array}{l}\text { superficial basal cell carcinoma, } \\
\text { actinic keratosis }\end{array}$ & Cryotherapy \\
\hline 8 & $\begin{array}{l}\text { Squamous cell } \\
\text { carcinoma/unknown }\end{array}$ & Excision & Squamous cell carcinoma & Excision \\
\hline 9 & Benign mole & Reassurance & Benign mole & Reassurance \\
\hline 10 & Benign mole & Reassurance & Benign mole & Reassurance \\
\hline 11 & Angioma & Reassurance & Angioma & Reassurance \\
\hline 12 & Atypical naevus & Excision & Atypical lesion-Lentigo maligna & Excision \\
\hline 13 & Benign mole & Reassurance & Benign mole & Reassurance \\
\hline 14 & $\begin{array}{l}\text { Unknown/squamous cell } \\
\text { carcinoma }\end{array}$ & Excision & Squamous cell carcinoma & Excision \\
\hline 15 & benign mole & Reassurance & Benign mole & Reassurance \\
\hline 16 & Unknown & Biopsy & Atypical pigmented lesion & Biopsy \\
\hline 17 & Melanoma & Excision & Melanoma & Excision \\
\hline 18 & Kaposi & Biopsy & Squamous cell carcinoma/Kaposi & Biopsy \\
\hline 19 & Lentigo & Reassurance & Lentigo & Reassurance \\
\hline 20 & $\begin{array}{l}\text { Squamous cell carcinoma/ } \\
\text { basal cell carcinoma }\end{array}$ & Excision & Basal cell carcinoma & Excision \\
\hline 21 & Basal cell carcinoma & Excision & Basal cell carcinoma & Excision \\
\hline 22 & Basal cell carcinoma & Excision & Basal cell carcinoma & Excision \\
\hline 23 & Actinic keratosis & Efudix cream & Actinic keratosis & $\begin{array}{l}\text { Moisturizer + topical } \\
\text { steroids }\end{array}$ \\
\hline 24 & Dermatitis & Moisturiser & Lichen sclerosus & Moisturiser \\
\hline 25 & Unknown & Examination & Dermatofibroma & Biopsy \\
\hline 26 & Benign mole & Reassurance & Benign mole & Reassurance \\
\hline 27 & Benign mole & Reassurance & Benign mole & Reassurance \\
\hline 28 & Unknown & To be seen & Blisters & Dressing \\
\hline 29 & Seborrheic keratosis & Reassurance & Seborrheic keratosis & Reassurance \\
\hline
\end{tabular}

this information efficiently and accurately for the consultant to consider. Therefore, ultimately, the diagnosis and treatment management options are decided upon by a medical expert. A limitation of the telemedicine solution is the focus on a single suspicious skin lesion and the potential increased risk of missing a skin cancer diagnosis in another area of the body (9). In a previous systematic review based on melanoma, Pala et al (10), concluded that teledermatology may be the answer to diagnosis, screening and management of skin cancers as well as pigmented skin lesions considering the increase demand for specialist care.

The present study compared this telemedicine solution with standard of care, (involving a full-body examination) and did not find other lesions that required excision. Patient willingness to use teledermatology in the future will be influenced by patient satisfaction. In a recent study based on a survey submitted by 184 patients, Hamad et al (11) described new patients reporting higher satisfaction with the teledermatology experiences compared to the satisfaction of existing patients in their clinic.

In conclusion, technology can improve the methods for medicine delivery. The DermaCheckup application exhibits a very good level of agreement in terms of diagnosis with traditional face-to-face appointments. In the present study, 93\% of patients could have been managed safely or referred straight for a biopsy, thus avoiding a visit to the hospital. A marked 
improvement in the efficiencies that can be potentially achieved was also noted, with the time required for patient management decreasing 46-22-fold, without considering the waiting time required from the time of organizing an appointment to the actual appointment. However, a risk associated with digital health care is that disparities in the patient access to dermatological services may arise, and this should not be overlooked.

\section{Acknowledgements}

Not applicable.

\section{Funding}

No funding was received.

\section{Availability of data and materials}

The datasets used and/or analysed during the current study are available from the corresponding author on reasonable request.

\section{Authors' contributions}

PCI, SS, JA, PG, JB and LS were involved in the design and conception of the platform, the study and in the reviewing of the manuscript. PCI, SS and LS contributed to the writing of the manuscript. PCI processed the image. All authors have read and approved the final manuscript. SS and PCI confirm the authenticity of all the raw data.

\section{Ethics approval and consent to participate}

In the present study, patient consent was obtained prior to the trial. Ethical approval has followed the local guidelines for clinical audits.

\section{Patient consent for publication}

Not applicable.

\section{Competing interests}

PCI, SS, JA, PG and JB were involved in developing the technological platform. JA, PG and JB are also affiliated with
L2S2 Ltd., the manufacturer of the teledermatology platform. LS has no competing interests to declare.

\section{References}

1. Triggle N: 10 charts that show why the NHS is in trouble. BBC News 2021 (online) Available at: https://www.bbc.co.uk/news/ health-42572110. Accessed March 15, 2021.

2. Ageuk: Later Life in the United Kingdom. 2019. http://www. ageuk.org.uk/globalassets/age-uk/documents/reports-and-publications/later_life_uk_factsheet.pdf. Accessed March 15, 2021.

3. Eedy D: Dermatology: A specialty in crisis. Clin Med (Lond) 15 509-510, 2015.

4. Giavina-Bianchi M, Santos AP and Cordioli E: Teledermatology reduces dermatology refferals and improves access to specialists. EClinicalMedicine 29-30: 100641, 2020.

5. O'Connor DM, Jew OS, Perman MJ, Castelo-Soccio LA Winston FK and McMahon PJ. Diagnostic accuracy of pediatric teledermatology using parent-submitted photographs: A randomized clinical trial. JAMA Dermatol 153: 1243-1248, 2017.

6. Yeboah CB, Harvey N, Krishnan R and Lipoff JB: The impact of COVID-19 on teledermatology: A review. Dermatol Clin 39: 599-608, 2021.

7. Assis Acurcio F, Guerra Jr AA, Marino Calvo MC, Nunes DH, Akerman M, Spinel LF, Garcia MM, Pereira RG, Costa Borysow ID, Silva RR, et al: Cost-minimization analysis of teledermatology versus conventional care in the Brazilian National Health System. J Comp Eff Res 10: 1159-1168, 2021.

8. Giavina-Bianchi M, Cordioli E and Dos Santos AP: Accuracy of deep neural network in triaging common skin diseases of primary care attention. Front Med (Lausanne) 8: 670300, 2021.

9. Elliott L and Sharma M: Teledermatology 2-week-wait skin cancer referrals during the COVID-19 pandemic: A service evaluation. Clin Exp Dermatol Sep 24, 2021 (Epub ahead of print).

10. Pala P, Bergler-Czop BS and Gwiżdż JM: Teledermatology: Idea, benefits and risks of modern age-a systematic review based on melanoma. Postepy Dermatol Alergol 37: 159-167, 2020.

11. Hamad J, Fox A, Kammire MS, Hollis AN and Khairat S. Evaluating the experiences of new and existing teledermatology patients during the COVID-19 pandemic: Cross-sectional survey study. JMIR Dermatol 4: e25999, 2021. International (CC BY-NC-ND 4.0) License. 